\title{
Géolinguistique
}

16 | 2016

Varia

\section{Lenticchie, semole e uova di tacchino: sui nomi delle lentiggini nei dialetti italiani}

Lentilles, semoule et oufs de dinde: sur les noms des taches de rousseur dans les dialectes italiens

Lentils, Groats and Turkey Eggs: On the Names of Freckles in Italian Dialects

Federica Cugno

\section{(2) OpenEdition}

\section{Edizione digitale}

URL: http://journals.openedition.org/geolinguistique/551

DOI: $10.4000 /$ geolinguistique.551

ISSN: 2650-8176

\section{Editore}

UGA Éditions/Université Grenoble Alpes

\section{Edizione cartacea}

Data di pubblicazione: 1 décembre 2016

Paginazione: 189-206

ISBN: 978-2-84310-342-7

ISSN: 0761-9081

\section{Notizia bibliografica digitale}

Federica Cugno, «Lenticchie, semole e uova di tacchino: sui nomi delle lentiggini nei dialetti italiani », Géolinguistique [Online], 16 | 2016, online dal 15 février 2019, consultato il 30 octobre 2020. URL http://journals.openedition.org/geolinguistique/551; DOI : https://doi.org/10.4000/geolinguistique. 551 


\title{
Lenticchie, semole e uova di tacchino: sui nomi delle lentiggini nei dialetti italiani
}

\author{
Federica Cugno \\ Università degli Studi di Torino
}

\section{Résumé}

L'article analyse du point de vue onomasiologique et motivationnel les différentes désignations dialectales italiennes relatives aux taches de rousseur, documentées par les atlas linguistiques nationaux et régionaux. Les domaines motivationnels les plus récurrents considèrent la forme et la couleur des taches de rousseur, avec des fréquentes associations métaphoriques avec des éléments de la culture matérielle.

\section{Mots-clés}

Géolinguistique, onomasiologie, dialectologie italienne, taches de rousseur.

\begin{abstract}
This article contains an onomasiological and motivational analysis of the various Italian dialectal terms for freckles found in national and regional atlases. Of all the terms discussed, the majority refer to the shape and the color of freckles, with frequent metaphorical associations with elements of folk culture.
\end{abstract}

\section{Keywords}

Linguistic geography, Onomasiology, Italian dialectology, freckles. 


\section{Le lentiggini negli atlanti linguistici}

I nomi dialettali italiani delle lentiggini, indicanti cioè quelle macchioline di colore scuro che compaiono specialmente sulla pelle delle persone con carnagione chiara, si distinguono, rispetto a altri lievi «difetti» cutanei come i nei, per la loro rilevante eterogeneità. Essi si prestano dunque ad essere studiati e analizzati sotto il profilo onomasiologico e motivazionale al fine di ricostruire sia gli aspetti della patologia risultati più significativi nel processo di lessicalizzazione sia l'immaginario popolare ad essi sottesi.

I dati linguistici su cui si fonda il presente studio provengono dalla carta 198 dell'Atlante Italo-Svizzero (AIS) le lentiggini e dalla carta 90 voglia di vino/neo dell'Atlante Linguistico Italiano (ALI), di cui lentigginiò costituisce una voce complementare, con le risposte presentate in forma di elenco. In quest'ultima opera le forme dialettali sono state raccolte in modo indiretto tramite un'illustrazione accompagnata dalla domanda «Come dite le macchiette che, come quella signorina, hanno specialmente quelli che sono di pelo rosso?». Ai dati collazionati su scala nazionale sono stati poi aggiunti quelli reperiti nell'Atlante Storico Linguistico Etnografico del Friuli (ASLEF, carta 312, vol. III), nell'Atlante Lessicale Toscano (ALT, dom. 412) e nell'Atlante Linguistico del Ladino Dolomitico II (ALD II, carta 49, vol. I). Benché in dermatologia si operi una distinzione tra le lentiggini, che sono permanenti, e le efelidi, che compaiono con l'esposizione al sole e tendono più o meno rapidamente a regredire in assenza di essa, tale differenziazione non è presa in considerazione nelle fonti consultate, i cui dati possono quindi riferirsi indifferentemente alle une o alle altre. Per ciò che concerne la produttività della domanda, ad esclusione della Sardegna, dove entrambi gli atlanti nazionali registrano risposte assai rade (4 nell'AIS e 16 nell'ALI) perché gli informatori mostrano difficoltà ad attribuire un nome al referente, in genere gli intervistati rispondono con prontezza e senza incertezze. Si dispone quindi di una documentazione abbastanza capillare relativa a tutto il territorio nazionale, che permette di individuare e eventualmente circoscrivere, nella pur ampia varietà di tipi lessicali, numerose aree omogenee di varia estensione ${ }^{1}$.

1. I dati provenienti dagli Atlanti sono stati resi in grafia fonetica secondo il sistema IPA, con una trascrizione semplificata rispetto a quella di partenza, mentre per quelli provenienti da altri testi si è conservata la grafia originale. 


\section{Continuatori diretti}

Per designare le lentiggini il latino disponeva dei termini lĕntīgo, - ̌nnis e lĕntīcula, ambedue attestati dal I secolo d.C. e diminutivi di lens, lĕntis «lenticchia» per la somiglianza tra le macchioline della pelle e la forma e il colore dei chicchi di questo legume (REW e REWS 4980 e 4981). Tuttavia, mentre lěntīgo, -l̆nis è impiegato esclusivamente nell'accezione di «lentiggine» a partire dalla sua prima attestazione nella Naturalis historia di Plinio il Vecchio, la voce lëntīcula è usata specialmente nel significato primario di «piccola lente; lenticchia», accanto a quello secondario di lentiggine rilevato per la prima volta in Celso nel suo trattato sulla medicina.

Tra i continuatori di lěntīgo,-ľnis, oltre all'italiano lentiggine (XIV sec.) (DEI 3, 2202; DELI 3, 663), figurano varie forme dialettali presenti soprattutto in Campania, Calabria e Sicilia e contraddistinte, come risulta dal confronto tra le attestazioni dell'AIS e quelle dell'ALI, da una progressiva espansione, sicuramente dovuta all'influenza della corrispondente voce italiana. Così in Campania, rispetto all'unica attestazione dell'AIS nel beneventano (Colle Sannita P. 714), l'ALI illustra una situazione in cui [lən' dinjə] è il tipo predominante nonché quello registrato nei principali vocabolari dialettali dall'Ottocento in poi (D'Ambra, 1873 [1969]; Andreoli, 1887; Altamura, 1956). L'area calabrese di [len'tijine], collocata in una ristretta fascia centrale della regione e documentata da due sole attestazioni dell'AIS (Melissa - KR P. 765 e Mangone - CS P. 761) risulta comprovata e meglio definita nell' ALI, comprendendo quattro località limitrofe del catanzarese (PP. 945, 947, 949, 944). Sulla base dei dati dell'ALI, anche in Sicilia il tipo lentiggini risulta maggioritario, con gli esiti [lin'tini], [lin'ti:ni] in cui la - $j$ - originatasi da - $g$ - spesso scompare senza lasciare traccia, secondo un modello riscontrabile anche in voci simili (cfr. gli esiti di impetigo, impetiginis «erpete» nella carta 683 dell'AIS). $\mathrm{Al}$ medesimo etimo va ricondotto anche l'abruzzese [rən'dindələ], forma reduplicata con rotacizzazione di $l$ - in $r$-, registrata dall'AIS nel chietino (Palmoli P. 658) con attestazioni più numerose nell'ALI in provincia di Campobasso (PP. 674, 675, 677) e di Foggia (P. 811) (REWS 4981; LEA).

Per ciò che concerne le aree settentrionale e mediana, le isolate attestazioni di lentiggini negli atlanti nazionali trovano conferma negli atlanti regionali, accanto ad ulteriori espansioni del termine, sotto l'influenza della lingua nazionale, in aree tradizionalmente contraddistinte da altri tipi lessicali. Così le inchieste dell'ALD II avvalorano la presenza del tipo lentìne/lintìne lungo la Val Camonica (a Breno, Darfo e Ponte di Legno), mentre dall'AIS e dall'ALI era riscontrato soltanto in forma isolata nel bresciano (AIS: Borno P. 238; ALI: Brescia P. 133), e lo circoscrivono ad 
una piccola area in cui, con tutta probabilità, la terminazione latina -iginem dà un esito -ina corrispondente a -ana dal latino -aginem, segnalato da Rohlfs per l'area bresciana e trentina con l'esempio di piantana da plantaginem (Rohlfs, 1966-1969) e riscontrabile anche nelle varietà retoromanze dell'Engadina (ALD II). Infine in Toscana il tipo lentiggini risulta maggioritario, sulla base delle recenti inchieste dell'ALT, nella provincia di Arezzo, come segnalato con isolate attestazioni dagli atlanti nazionali (l'AIS a Cortona, l'ALI a Foiano della Chiana e a Cortona), e non, come si poteva attendere, nel fiorentino, che, come si vedrà, gli preferisce crusca.

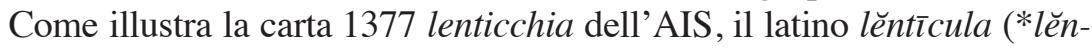
ťcula) è continuato diffusamente nei dialetti italiani nel significato botanico, rispetto al quale quello secondario di lentiggini si presenta sempre in unione con il precedente, ma in forma più circoscritta. Infatti i continuatori di lĕntīcula (*lĕntı̆cula) nella sua accezione medica ${ }^{2}$ contraddistinguono nell'Italia settentrionale un' ampia area occidentale che si estende dalla Valle d'Aosta ([lənt'i i əə]) al Piemonte ([lan'tije], [lent'itf], [lan'tidzi]), comprendendo anche le parlate occitane e francoprovenzali, fino alle regioni limitrofe della Lombardia ([lan'tiffe], [lan'tedze]) e della Liguria. Una seconda area solamente accennata dalle inchieste degli atlanti nazionali ma ben definita nell'ALD II è quella relativa alle parlate ladino-dolomitiche contrassegnate dalla scomparsa di $l$ - iniziale confuso con l'articolo e da vari esiti locali del nesso - $c l$-: nel gardenese (PP. 8687 e 88) il primo elemento del nesso passa da velare ad apicodentale ([ant' idlas]); nel fodom (PP. 94, 95, 96) si conserva il tratto velare, seppure sonorizzato ([an'tigles]); in Val di Fassa si riscontra la palatalizzazione del nesso ([ant'iKas]: PP. 97-100) fino alla sua scomparsa in val Badia ([an'ties]/[a'ntis]: PP. 81-85; 89-91) ${ }^{3}$. Altre attestazioni del tipo lenticchie figurano poi nell'Italia centrale in alcune località dell'Umbria meridionale ([lend' icce]), in Abruzzo e, in forma più sporadica, nel Lazio, dove comunque è la variante registrata a Roma ([lent'icce]); infine nell'Italia meridionale se ne riscontrano testimonianze in Campania, ma specialmente in Calabria ([lent'icce] [lint'icci] f. pl.), dove è il tipo maggioritario, e nell'estremità nord orientale della Sicilia.

2. Continuata anche nel francese lentille, nell'occitano lentilha, nel catalano llentía, nello spagnolo lenteja e nel portoghese lentilha.

3. Come si vedrà in seguito l'ampezzano e il ladino cadorino presentano invece il tipo ['lintimes]/['lintime] (PP. 92, 93). 


\section{Le altre denominazioni}

Le denominazioni che non continuano le forme latine fanno per lo più riferimento alle caratteristiche più significative delle lentiggini, ovvero alla loro forma e al loro colore. Tra queste si distingue un consistente gruppo di termini che trae origine da associazioni metaforiche con elementi della cultura materiale. Infine si distinguono alcune voci che appartengono all'area semantica delle eruzioni cutanee, designando in generale delle vere proprie affezioni come l'impetigine e l'herpes.

\subsection{Denominazioni legate alla forma}

Considerato che le lentiggini o le efelidi si manifestano sotto forma di piccole macchie sulla pelle, in molte parlate esse sono designate con termini il cui significato originario o attuale è macchia. Tra questi figura il tipo pane, forma femminile del lat. pannus «panno» (REW e REWS 6204) continuata specialmente nell'Italia settentrionale con il significato, presente anche nell'italiano antico, di macchia, chiazza della pelle, brufolo, pustola. Il lessotipo occupa un'area abbastanza uniforme che ha il suo epicentro nel Veneto irradiandosi nelle limitrofe parlate lombardo-orientali, dove però prevale il tipo maschile [p'ani] pl., e in quelle emiliano-romagnole. Dall'ALD II si evince che tale espressione è diffusa anche nell'area più orientale del Trentino (con esclusione delle località ladine) in contiguità con le parlate più meridionali della provincia di Belluno $\mathrm{e}$, a seguire, a sud, specialmente nelle province di Vicenza e Treviso, dove costituisce il tipo più usuale; invece le attestazioni nel Trentino occidentale, quasi interamente occupato dal tipo [lent], sono sporadiche e spesso contrassegnate come arcaiche. Le varietà delle province di Sondrio e Bergamo presentano le forme derivate [p'anule] f. o [p'anuli] m. (ALI: PP. 108, 122; ALD II: PP. 13-34), o provenienti direttamente dal latino pannŭlus «panno» (REW 6203) per la forma di piccolo lembo sulla pelle (Bianchini \& Bracchi, 2003; REW 6204; DEI 4, 2750).

Sulla base delle sporadiche attestazioni degli atlanti nazionali e soprattutto a partire dai dati raccolti dall'ALD II il tipo ['take] pl. (PP. 105, 104, 153), proveniente dal latino *tacca $(\mathrm{m})$, a sua volta mutuato dal germanico taikn "segno divino, prodigio» (REW 8534), insieme ai diminutivi ['takoles] (P. 136), [tako'lina] (P. 131) e [tatfa'dina] (PP. 129 e 130) e all'aggettivo [tako'lada] lentigginosa (PP. 143, 144, 145, 149, 150), pare concentrato in alcune varietà del trentino orientale e nella zona venetoladina del bellunese.

La medesima categoria motivazionale è all'origine della voce nuorese ['mantsaza], plurale di mantsa «macchia», e della locuzione cagliaritana 
[est amrat' tfada] lett. «è macchiata» da ammančai «macchiare», entrambe riconducibili al castigliano mancha (DES).

Infine, nella località galloitalica di Sperlinga (EN) (AIS: P. 836) figura il tipo ['berbəzi] pl., voce riconducibile al germanico derbita «macchia» (FEW $3,46 b$ ) con assimilazione $d / b$ e assai diffusa nell'Italia settentrionale per «erpete» (cfr. AIS: carta 683 erpete).

\subsection{Denominazioni legate al colore}

Alcune voci riflettono un collegamento al colore delle lentiggini o all'aspetto chiazzato o maculato del viso lentigginoso. La prima motivazione è riconoscibile nel termine [l'uvri] $\mathrm{m}$. pl., proveniente dal latino rubrum «rosso» (NDDC), la cui unica attestazione presente nell'AIS (P. 772, Centrache $\mathrm{CZ}$ ) è confermata dai dati dell'ALI, grazie ai quali si evince che è voce caratteristica di una piccola area del catanzarese corrispondente alle località di Girifalco, Chiaravalle Centrale, Badolato e Monasterace, quest'ultima in provincia di Reggio Calabria.

Tra i continuatori del latino tardo picula, diminutivo di pix «pece» vanno annoverate le forme ['petfa]/['pekjkja] lentiggini registrate dall'ALT in Lunigiana (Fivizzano, Vinca e Casola) e infine il chietino ['peccə] (ALI: PP. 672) che in Abruzzo è comunemente impiegato con il significato di chiazza (LEA).

Rientra ancora tra i termini legati al colore il tipo romagnolo [al 'gadzi] di San Pietro in Vincoli (ALI: P. 450) ${ }^{4}$ collegato a gagg «rossiccio di pelo rosso/lentigginoso», il cui etimo coincide con quello dell'italiano antico gazzo propriamente «di colore verde turchino come le piume della gazza» con successivo passaggio ad indicare «chi, oltre agli occhi azzurri, ha i capelli biondi» e infine il colore «biondo-rossiccio» (Miniati, 2010).

A entrambi gli ambiti motivazionali possono essere ricondotte le espressioni piemontesi [le dzaju'la] (ALI: P. 50) e [e dzaju'ra] (AIS: P. 167) lett. «è lentigginoso»e il sostantivo ['dzaje] lentiggini (AIS: P. 156). Seguendo il REP, infatti, si tratterebbe di termini appartenenti a una famiglia di origine galloromanza in cui confluiscono, con significati di tipo cromatico dal nero al variopinto, variegato voci probabilmente di origine diversa. Quindi per ['dzaje] si ipotizza un prestito dal francese jais «giaietto, varietà di lignite» derivato dal laino gagātes (dal gr. gagatés «pietra nera di Gages»), passato poi a designare una qualsiasi pietra o perlina di colore nero, mentre per l'aggettivo [dzaju' la] si chiama in causa anche il latino *găllium «variopinto, chiazzato, screziato».

4. Per la quale si veda anche gàgia «efelide, lentiggine» in Ercolani (1994). 
In una vasta area che occupa gran parte della Puglia e della Basilicata con propaggini nella Calabria meridionale figura il tipo ['perce] pl. proveniente dal latino *pĕrcŭla, da ricondurre, con tutta probabilità, al greco $\pi \dot{\varepsilon} \rho \kappa o \varsigma$ «macchiettato di nero», da cui il neogreco $\pi \varepsilon \rho \kappa \nu \alpha ́ \delta \alpha$ lentiggine rossore (Brighenti, 1927), e alla base anche dell'antico italiano perca «specie di pesce maculato» (REW e REWS 6401).

Il riferimento all'aspetto «pitturato, macchiato» è alla base del tipo [pent'elle] (REW, REWS 6512), deverbale del lat. pingere, raccolto dall'ALI in tre località marchigiane in provincia di Ancona e Macerata (PP. 470, 478 e 480), mentre la forma sarda [e pin'tada] lett. «è dipinta, macchiata» di Villacidro (P. 767) può essere ricondotta a pintare «pingere, dipingere» da *pinctare.

\subsection{Denominazioni metaforiche}

Come si è detto un cospicuo numero di nomi dialettali trae origine da associazioni metaforiche operate dal parlante tra l'aspetto delle lentiggini e vari elementi della cultura materiale, che sono stati raggruppati nelle seguenti categorie: legumi, cereali e altri vegetali; crusca; uova di tacchina; escrementi; piccoli insetti; gocce.

\subsubsection{Legumi, cereali e altri vegetali}

Alcune denominazioni rimandano a termini designanti legumi, cereali e altri vegetali i cui chicchi o bacche, per la forma tondeggiante o il colore scuro o rossastro, sono accostabili alle lentiggini, secondo un'associazione metaforica già produttiva, come si è visto, in latino (REW 4979).

Da latino lens, lentis «lenticchia» continuano forme femminili attestate in tre diverse aree della penisola italiana, dove si mantiene anche il significato originario di ambito botanico (cfr. carta 1377 lenticchia dell'AIS e i dati dell'ALI alla voce 3788 ervum lens - lente). La prima, contraddistinta dalla forma [le lent], è situata nel Trentino occidentale e centrale, dove, sulla base dei dati dell'ALD II, appare ancora vitale e maggioritaria. La seconda si colloca in Friuli, dove i singolari collettivi [lints], [lins] e [lens] risultano essere le varianti prevalenti, con una più intensa concentrazione nel friulano centrale. La terza area, tracciabile sulla scorta dei dati dell'AIS e dell'ALI e contrassegnata dalla forma ['lenda], anche qui un singolare collettivo, si sviluppa lungo il confine tra Marche e Abruzzo, interessando le province di Macerata, Pesaro-Urbino, Rieti, Ascoli Piceno, Teramo e Pescara.

Nell'ALT, in tre località contigue situate al confine tra le province di Firenze e Pistoia (Chiesina Uzzanese, Monsummano e Cerreto Guidi), figura il termine [sa'ina], forma regionale toscana per «sorgo, saggina», dal lat. 
$\operatorname{sagina}(m)$ «ingrasso, nutrimento degli animali, pastura» (EVLI), non registrato dagli atlanti nazionali e sconosciuto dagli informatori più giovani.

La forma [pibi'rinu], riscontrata in varie località nella provincia di Sassari e a S. Lussurgiu (CA) dall'ALI, ma assente nell'AIS, è da ricondurre al verbo pibirinare «picchiettare con macchie quasi di pepe»,

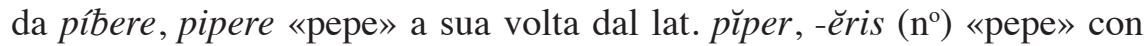
l'aggiunta del suffisso diminutivo -inus (DES).

Il tipo coccola, riconducibile a *coccula, forma diminutiva del lat. cŏccum «nocciolo della frutta» che indica le bacche e in particolare quelle del ginepro (DAM), è presente con sporadiche attestazioni nell'ALI, a Rionero - PZ (P. 902) e a Villa Canale - CH (P. 673), qui nella variante metatetica e con $s$-estrattivo [skə'lokkə], e nell'AIS a Roccasicura - IS (P. 666).

\subsection{2. crusca}

Un gruppo numericamente rilevante di termini dialettali indicanti la crusca, ovvero il residuo della macinazione del grano, viene impiegato in diverse aree della penisola anche nell'accezione secondaria di lentiggine, sulla base della somiglianza tra il colore e la forma delle scagliette del cereale. Si tratta, per l'area ligure, della voce ['brenu] crusca e lentiggine, derivata della base preromana *brenno «crusca» (REW 1284; FEW *brenno) e attestata dall'ALT anche a Capraia (Livorno), dal momento che l'isola fu a lungo possedimento genovese e fece parte della provincia di Genova fino al 1925. Dalla parallela forma femminile *brenna hanno origine le varianti campane e basso laziali ['vrennə] crusca e lentiggini, con numerose attestazioni dell'ALI a fronte dell'unica testimonianza riportata dall'AIS (P. 648 Fara San Martino - CH).

In area emiliano-romagnola, con propaggini anche nelle Marche settentrionali, troviamo l'impiego del termine ['remal], propriamente «crusca», dal latino *rĕmŏlum «crusca», da *remŏlĕre «macinare di nuovo». Dalle attestazioni dell'ALT il lessotipo risulta presente anche nella varietà della Lunigiana (a Pontremoli, Filetto, Fivizzano in provincia di Massa), e in una località limitrofa al territorio marchigiano (Marradi).

La voce di origine germanica crusca (da *krūska «crusca, farina» REW 4788), diffusa in questa accezione specialmente in Lombardia e in Toscana, figura anche con il significato di lentiggini soltanto in Toscana, in un'area circoscritta alla provincia di Firenze, dove compare talvolta anche la variante più rustica ['fruska], con alcune incursioni, come documenta l'ALT, nelle località più contigue delle limitrofe province di Arezzo e Prato.

Nell'Italia centrale, ovvero in gran parte della Toscana (con l'eccezione della provincia di Firenze), nel Lazio centro-settentrionale, in Umbria e 
nelle Marche prevalgono le forme ['sembola], ['semmole] ['sembole] provenienti dal latino * simŭla $(m)$, per il latino classico sümŭla $(m)$ «fior di farina» (REW 7806), impiegato comunemente in tali zone per designare la crusca (AIS carta 257 crusca; ALI voce 965). Dagli atlanti regionali si ricavano ulteriori sporadiche attestazioni del lessotipo in Veneto (ALD II P. 141: ['semole]) e in Friuli (ASLEF PP. 42, 51, 213, 211: ['semole]).

In un' area del Salento compresa tra Brindisi e Lecce, con saltuarie attestazioni che giungono fino al foggiano (ALI: PP. 803 e 807), è diffusa la voce [ka'nigjgja], propriamente «crusca», proveniente dal lat. *canīlia «crusca» ovvero «ciò che si dà ai cani» (REW, REWS 1589).

Infine rientra nel gruppo di denominazioni designanti la crusca anche il tipo sardo [ $\mathrm{t} f i v r a ' z e d d u]$ «cruschello fine», derivato di [ $\mathrm{t} f \mathrm{i}^{\prime}$ vrazu] «cruschello» a sua volta proveniente dal latino (panis) cibarius (REW 1895; DES).

\subsubsection{Uova di tacchina}

Il tipo uova di tacchina scaturisce dall'aspetto picchiettato delle uova di questo gallinaceo e risulta caratteristico di alcune parlate dell'Italia nord orientale. Nello specifico in friulano figurano i tipi ['wovi/ows de 'dindja] e ['vwovi de 'puja] (ASLEF: P. 121 Vigonovo) mentre in Veneto, specialmente nelle province di Venezia e Pordenone, si trova la variante ['vovi de pi'tona], documentata anche dall'ALT in Toscana, ad Alberese, località della Maremma interessata negli anni Trenta da un consistente flusso migratorio di contadini di origine veneta. È probabile che questo tipo lessicale conoscesse un tempo una diffusione areale maggiore di quella attuale; ne è prova, da un lato, la sua presenza, seppure isolata, in Lombardia (ALI: P. 152) e dall'altro l'esistenza di espressioni simili come ['matfa da 'pola] macchie di tacchina nel bresciano (ALI: P. 267), ['toki] e [tu'kî] lett. tacchino in provincia di Modena (ALI: PP. 408 Migliarina e 409 Quarantoli) e bille a Lucignano in Val di Chiana in provincia di Arezzo (ALT: P. 168) dove il tacchino è detto appunto billo (cfr. ALT: dom. 190e). In queste ultime località si è probabilmente persa la motivazione di partenza legata all'aspetto delle uova e si è conservato solo il riferimento al tacchino.

Meno chiare sotto il profilo della motivazione risultano invece le espressioni ['ovi de 'ruza] e ['vwovi de 'ruja] propriamente uova di bruco raccolte dall'ALD II in provincia di Treviso rispettivamente ai PP. 162 e 193 (cfr. carta ALD II 748 il bruco/la lucciola); trattandosi di località contigue a quelle contraddistinte dal tipo uova di tacchino, potrebbe trattarsi di una rimotivazione della locuzione non più basata sull'aspetto delle uova, bensì sulla loro dimensione. 


\subsubsection{Escrementi}

Una motivazione che ricorre, seppure in forma isolata, in varie zone dell'Italia, si basa sulla somiglianza delle lentiggini con gli escrementi. Figurano dunque le forme ['mert] pl. (P. 145) e la locuzione ['merd ed 'musk] pl. merde di mosche (P. 148) raccolte dall'ALI in Lombardia, le espressioni [ke' gade de 'moske] in Veneto (P. 147) in provincia di Belluno e [ka'gadis de 'mostfis] in Friuli (P. 202), in provincia di Pordenone, registrate dall'ALD II e infine la forma [ka'gadis di 'mosce] presente in Friuli in provincia di Udine (ASLEF: P. 131 Manzano e P. 100a Ceresetto). Ulteriori esempi della produttività di tale immagine sono offerti da [ $\mathrm{k}$ ka'gadłdze], registrato dall'ALI presso i tabarchini, da ricondurre al ligure scagàgia letame, il bresciano ['zakle] (AIS: P. 249 e ALDII: P. 36) cioè «caccole, sterco che si attacca alla lana della pecora» (cfr. AIS, carta 1083 caccola P. 259 e ALI, voce 4301 caccola, zeccola) e infine la locuzione schit d'eva letteralmente «escremento di ape» documentata in Val Gardena (Forni).

\subsubsection{Piccoli insetti}

In Calabria in due località contigue della costa ionica catanzarese (ALI: PP. 933 e 938) si trova il tipo [a 'tyimi' tyia], con tutta probabilità un derivato di cimice, che più a nord assume il significato di «noia, molestia, irrequietezza, travaglio» (Battipede, 1987, s.v. cimicìa), ovvero la condizione di chi è assalito dalle cimici.

Potrebbe forse rientrare in questa categoria il tipo lessicale ['rollə] [rullə] f. pl. raccolto dall'ALI in provincia di Campobasso (PP. 687, 688, 693 e 694 - Torella del Sannio, Cercemaggiore, Campodipietra, Boiano) e non attestato nell'AIS. Se infatti nel LEA la voce, nella variante rolla, rimane senza spiegazione etimologica e ben distinta dall'omografo ['rollə] porcile ricondotto al latino (ha)rŭlla diminutivo di hara «porcile», la variante rélla di Campobasso viene invece lemmatizzata sotto rélle, che in aquilano indica le zecche.

\subsubsection{Gocce (di pioggia)}

L'accostamento delle lentiggini a delle gocce è sottesa all'espressione [est agutt' ara]/[est agut'tada] registrata dall'ALI nel nuorese e nel cagliaritano e riconducibile a gutta «gotta, gocciola» (DES). La medesima immagine figura nell'estremità nord orientale della penisola, dove l'ALI e specialmente l'ASLEF riportano attestazioni del tipo [zbi'vitfis]/[pi'viffis] forma pl. di sbivicie «acquerugiola, assai rada e minuta» (Pirona, 1935). 


\subsection{Alcune denominazioni delle lentiggini rientrano nell'ambito semantico delle affezioni della pelle come l'impetigine, l'herpes o più in generale le pustole}

\subsubsection{Impetigine}

Dal latino impetīgo, - ̌nis erpete, un deverbale da impetěre attaccare (REW e REWS 4306), derivano forme dialettali che in alcune aree hanno assunto il significato specifico di lentiggine accanto a quello più diffuso di erpete e eruzione cutanea (cfr. carta 683 erpete dell'AIS). La prima zona di diffusione è la Toscana, come ben documentano le attestazioni dell'ALT che precisano quelle assai più sporadiche dell'AIS e dell'ALI. Il tipo, declinato nelle varianti pitiggini, petiggine, pitìgine, putìggini, e nella forma aferetica tiggine (quest'ultima a Volterra e a Montecatini Val di Cecina) è attestato specialmente nelle province di Lucca (Brandeglio e Pieve Fosciana, Massarosa, Porcari, Pescaglia, Piazzano, Marlia) e di Siena (Valiano, Montepulciano e Torrita Stazione, Montallese) e in località ad esse limitrofe (Antona - MS).

Incrociando le informazioni sulla vitalità del lessotipo con le caratteristiche sociolinguistiche degli informatori si evince che si tratta di una forma in recessione, generalmente riferita dai soggetti più anziani e conosciuta passivamente da gran parte degli altri. Più a sud l'AIS la documenta ancora nel Lazio meridionale (PP. 664 e 682), più diffusamente in Campania, dove è anche il tipo registrato nel capoluogo, e nell'estremità meridionale del Salento ([pi'tifina]); assai meno frequenti sono invece le attestazioni dell'ALI con due sole testimonianze in Campania, mentre altre figurano nel nord della Puglia, più a sud nel barese e in varie località della Basilicata sia del centro sia della parte più occidentale della regione. Per ciò che concerne la Calabria la forma [pu'tihini] registrata dall'ALI a Vibo Valentia (CZ) e a Giffone (RC) (PP. 959 e 953) conferma quella dell'AIS a Serrastretta $(\mathrm{CZ})$.

La forma [pal'ledzdzina], registrata dall'ALI in Toscana in una località della provincia di Arezzo (Bibbiena P. 525), alla luce dei dati dell'ALT risulta effettivamente un tipo circoscritto ad una piccola area dell'aretino (Campi di Bibbiena, Badia Prataglia e Chiusi della Verna) e può ragionevolmente essere spiegata come un incrocio tra i tipi lentiggine e impetiggine, ambedue presenti nella regione.

Al medesimo campo semantico appartiene l'espressione di area settentrionale volatiche attestata dall'ALI a Padova (P. 272) e dall'ALD II in Trentino (PP. 115 e 116, due località confinanti con il Veneto). Le testimonianze dell'AIS si collocano invece più a sud, in Emilia Romagna, precisamente a Bologna [el vu'ladk] e, nella variante con specificatore [vu'ladek 
dal sol], in provincia di Modena a Nonantola e a Concordia. Come si evince dai dati della carta 683 erpete dell'AIS, il lessotipo, proveniente dal latino volatǐcus «che vola», a sua volta derivato da volāre (REW 9432), è diffuso con tale significato in una vasta area che comprende Emilia Romagna e Veneto.

$\mathrm{Al}$ medesimo ambito motivazionale appartiene la voce ['Jkjafane] pl. registrata dall'ALI in Umbria (Umbertide - PG) e dall'AIS sulla costa adriatica marchigiana (Sant'Elpidio a Mare - FM e Ancona). Si tratta di un termine diffuso in Italia centrale per indicare l' «herpes» (cfr. AIS IV 683 'erpete') derivato da šchiafə f. termine medico che designa vari tipi di affezioni cutanee (LEA).

Va segnalato infine il tipo ['tserras] raccolto a Isili (NU) e proveniente dal lat. tardo zerna, accanto a serna e sarna, voci di origine preindeuropea che hanno il significato generico di erpete, eczema (DES sv. Aerra).

\subsubsection{Petecchia}

L'uso del tipo petecchia, dal latino tardo *(im)petĭcŭla $(m)$, forma diminutiva di impetīgo, - ̌nnis erpete, è peculiare di alcune varietà bresciane ([pe'tedze]), come documentano sia gli atlanti nazionali (AIS: PP. 259 e 254; ALI: PP. 118, 140, 134) sia l'ALD II (PP. 41, 42, 43 e 46). Il lessotipo figura anche in attestazioni isolate raccolte in Sicilia e Calabria (ALI: PP. 1039 e 962) dove in genere assume vari significati sempre relativi a macchie, cicatrici (NDDC, VS). La voce petecchia, ricordata come forma tradizionale da più informatori ma in graduale disuso, figura infine come unica attestazione nell'ALT a San Pellegrino in Alpe, frazione divisa tra i comuni di Castiglione di Garfagnana e di Frassinoro e tra la provincia di Lucca e quella di Modena.

\subsubsection{Pustola/Pustole}

In varie località le denominazioni fornite per lentiggini corrispondono a quelle designanti la pustola. Ad esempio il tipo frivuliti pl., attestato in due località rispettivamente del barese e del tarantino (PP. 829 e 866), significa propriamente «bollicine fitte e diffuse sulla pelle per sudore, sudamini» (VDS). E ancora l'ALI raccoglie a Daiano (TN) il tipo ['pabje] f. pl., dal latino papula; in Sardegna figura il tipo [pa'bedda] «pustola» e nome di varie malattie pustolose, specialmente del vaiolo, proveniente dal lat. papilla (REW 6212) o da papula (REW 6215) con altro suffisso (DES). Invece la voce [pi' gotta], prestito dal catalano pigota, pigoni da piga «lentiggine», è attestata nel cagliaritano (Cagliari; Sarrocco) dove, secondo il DES, la sua fortuna era facilitata dall'esistenza del verbo pigaj nel significato di attecchire e di pigoni petecchia e anche, isolatamente, 
vaiolo o morbillo. La stessa immagine è all'origine del padovano ['tavare] di Campo San Martino (PD), voce che indica una «piccola enfiatura per lo più da morsicatura di zanzara, vespa, ortiche e simili» (Boerio, 1856 ${ }^{2}$ ), dalla medesima base etimologica di tavarà, participio passato e aggettivo dal significato di «macchiettato, picchiettato» registrato nel Dizionario veneziano della lingua e della cultura popolare nel XVI secolo (Cortelazzo, 2007). La forma [tsi 'trilə] raccolta dall'AIS a Saracena (CS) (P. 752) significa propriamente «foruncoletto, pustoletta» (NDDC zitrile). Il sardo [Bißi'zia] assume in varie località (Ploaghe, Macomer, Norbello e Làconi) il significato di «bolla, pustoletta»e in campidanese quelli di «spillo, foro fatto nelle botti» (cfr. AIS, carta 686 pustola).

\subsection{Credenze popolari}

La documentazione dei due atlanti nazionali testimonia la sporadica esistenza in Sicilia del tipo [sa'vuku] un singolare collettivo (VS) dal latino $\operatorname{sab} \bar{u} c u(m)$, «sambuco», limitato alle province siciliane di Enna e Caltanissetta nell'ALI (ALI 1037 e 1050), ma attestato dall'AIS anche a Baucina $(\mathrm{PA})$, a San Biagio Platani (AG) e nella località galloitalica di Aidone (qui nella variante [sa'mu] da sambucus). La presenza di tale lessotipo può essere legata alle prescrizioni della medicina popolare siciliana che raccomandano di usare il sambuco per eliminare le lentiggini e, nel contempo, alle credenze popolari secondo cui l'apparizione delle lentiggini sarebbe influenzata dalla fioritura e dal ciclo vegetale del sambuco, pertanto le macchie apparirebbero quando il sambuco fiorisce e sparirebbero o si attenuerebbero alla nascita delle foglie o alla loro caduta (Pitré, 1896: 223). Considerata la produttività della categoria motivazionale basata sulla somiglianza ravvisata tra le lentiggini e vari tipi di vegetali, non si può escludere che anche questo lessotipo sia da ricondurre a questo ambito sulla scorta dell'aspetto delle bacche del sambuco. È noto infatti che una delle peculiarità della medicina popolare è quella di confidare, per le indicazioni terapeutiche di un'affezione, su elementi che abbiano qualcosa in comune con essa; può dunque accadere che non sia il nome che dipenda dalla terapia, ma che la seconda sia stata suggerita dal primo.

\subsection{Voci di incerta spiegazione}

La voce [la frint], raccolta dall'ALI soltanto a Collina (UD), è confermata, seppure come arcaismo, nella medesima località dall' ALD II (P. 195), mentre nell'AIS figura a Forni Avoltri (P. 318) e a Cedarchis (P. 319). Nell'atlante friulano, dove si avverte che frint è un singolare collettivo, si hanno attestazioni più frequenti concentrate sempre nel nord della regione, 
in particolare in Carnia, lungo la valle del But da Paluzza a Tolmezzo (qui nella variante frunt), e in Val Degano. Poiché il termine, riconducibile al latino frons, fronde, in friulano indica propriamente le fronde, ovvero ramoscelli d'albero raccolti verdi le cui foglie servono di cibo alle capre e alle pecore, e, secche, servono anche di letto agli armenti (Pirona, 1935), in assenza di informazioni su eventuali usi terapeutici o credenze popolari, vi si potrebbe forse scorgere un accostamento con le lentiggini motivato dall'aspetto degli arbusti.

Il tipo ['lintimes]/['lintimas], sulla base dell'insieme delle testimonianze dell'AIS, dell'ALI, dell'ASLEF e dell'ALD II risulta contraddistinguere le parlate ampezzane e cadorine del Bellunese (AIS: Zuel P. 316; ALD II: PP. 92 Cortina d'Ampezzo e 93 Colle S. Lucia e PP. 132 Lorenzago, 133 Pozzale, 134 Cibiana, 135 Vinigo (Vodo), 137 Selva, 138 Rocca Pietore), alcune altre varietà limitrofe della provincia di Pordenone con caratteristiche linguistiche intermedie al friulano ed al ladino (ASLEF: PP. 39 Cimolais, 38 Erto; ALD II Cimolais 203 e Erto 204) e infine altre parlate situate più a est, in provincia di Udine (ASLEF: P. 42 Tramonti di Sotto, PP. 15 Dogna (Pellis) e 21a Raccolana; ALD II: P. 198 Forni di Sopra; AIS: Forni di sotto UD P. 327, Tramonti di Sotto P. 328). La voce lèntima, attestata in italiano nel XVII secolo con il significato di «fodera del guanciale o del materasso», è ricondotta al latino ı̌ntı̆ma, a sua volta dal greco éndyma «veste», ovvero un grecismo dell'Esarcato di Ravenna raccostato per paretimologia al lat. intimus «intimo» (DEI), con successiva concrezione dell'articolo. Considerando che le contigue varietà ladine dolomitiche, come si è visto, presentano in veste compatta dei continuatori del latino lěntīcula, si può ipotizzare che il tipo lintimas, nasca da un uso metaforico della voce lintima a partire dall'aspetto picchiettato della fodera dei pagliericci. Infatti, come testimoniano i vocabolari dialettali, in area bellunese la voce è attualmente impiegata al singolare per designare «tessuto per federe» (Menegus Tamburin, 1973 s.v. lintima), «traliccio» e «tessuto di tela per materassi e guanciali» (Menegus Tamburin, 1978) e un tempo probabilmente ha conosciuto una diffusione maggiore, anche in Friuli Venezia Giulia, come riportano le attestazioni raccolte dall'ALI alla voce $\mathrm{n}^{\circ}$ 1066bis «fodera del saccone» a Mereto di Tomba (UD), Grado e Trieste (PP. 326, 354, 357).

\section{Conclusioni}

A conclusione di questa breve rassegna, si può rilevare che la maggior parte dei termini dialettali italiani designanti le lentiggini può essere facilmente ricondotta ad una delle categorie motivazionali individuate, tra le 
quali spicca per la frequenza di attestazioni quella che associa le lentiggini alla crusca. Essa infatti, espressa nei vari termini locali designanti la crusca (brenu, remolo, crusca, semola, vrenna, caniggia) interessa gran parte dell'Italia centrale, configurandosi come una innovazione che, stando all'area di diffusione, potrebbe avere avuto il suo fulcro nella Toscana. A nord di quest'area figura a occidente un'ampia zona con i continuatori di lěntīculam, che si pone in continuità con il territorio galloromanzo, mentre più a est spicca l'area lent frammentata in due tronconi dall'espansione del tipo veneto pane. L'Italia meridionale invece, oltre a mostrare vaste zone in cui continuano i tipi latini, si distingue per la presenza diffusa del grecismo perchia in Puglia e Basilicata. La lettura complementare di più fonti e in particolare degli atlanti regionali ha permesso poi di delineare, all'interno del quadro generale appena descritto, piccole aree lessicali contraddistinte da voci, che, per la loro attestazione episodica in una delle fonti considerate, parevano suggerire una dimensione esclusivamente idiolettale. È il caso del tipo ladino cadorino e friulano lintimes/lintimas, del friulano frint, della locuzione veneta «uova di tacchina», del toscano saina, del molisano rolle attestato in provincia di Campobasso e del calabrese luvri circoscritto a poche località del catanzarese.

Sul piano semantico la presenza di un numero rilevante di termini connotati in senso negativo, sembrerebbe riflettere una preclusione popolare nei confronti delle lentiggini. La loro designazione con voci indicanti vere e proprie malattie della pelle, escrementi e una varietà scadente di grano come il sorgo, per giungere infine alla crusca, ovvero al prodotto meno nobile del grano, pur sulla scorta di eventuali connessioni suggerite dal suo colore e dalle sue dimensioni, avvalorano l'idea che le lentiggini siano considerate nell'immaginario popolare un difetto o un'imperfezione della pelle che contrasta con il tradizionale ideale di bellezza rappresentato da un incarnato chiaro e non toccato dal sole. Tale pregiudizio, che traspare nel commento [a sa' jejva 'bela ma le 'pinna de len'tigje] «sarebbe bella, ma è piena di lentiggini» riportato dall'AIS a Noli (AIS: P. 185 n.), e forse anche associato a quelli ricorrenti fin dall'antichità nei confronti delle persone dai capelli rossi, è confermato dalla varietà e dalla quantità di rimedi popolari per attenuare o eliminare le lentiggini sedimentatisi nella medicina popolare e tramandatisi fino ai giorni nostri.

Ad esempio, oltre a quanto documentato da Pitré per la Sicilia in merito all'uso del sambuco, in Piemonte, a Voghera, le lentiggini si eliminavano bagnandole in primavera con la linfa delle viti (Maragliano, 1962); in Toscana si lavavano con urina, con latte di fico, o con acqua in cui era state cotte quindici uova, oppure venivano unte con succo di cipolle o spalmate con corallo disciolto in succo di limone (Pazzini, 1948); in Friuli, come 
riferisce un informatore di Tricesimo (AIS: P. 338 n.), si usava lavare il viso con la linfa della vite, oppure, come riferisce Pazzini (1948), con acqua delle viti potate o con acqua in cui siano state immerse galle di quercia o prezzemolo; dalla medesima fonte si apprende anche l'esistenza di una cura magica consistente nel guardare ogni notte la luna durante l'intero corso facendo il gesto di pulirsi delle lentiggini, che, in tal modo, a luna nuova, dovrebbero scomparire; in Sardegna per eliminare le lentiggini vi si spalmava sangue di pipistrello ${ }^{5}$. A questi rimedi si possono poi aggiungere i seguenti, registrati nel Vocabolario della Crusca a partire dalla terza edizione dell'Opera (Crusca, 1863-1923): la biacca e la canfora, l'acqua e' lisci, la cocitura dei lupini, l'olio del seme del lino selvatico (s.v. lentiggine), la rugiada raccolta sulle foglie del cavolo (s.v. lentigginoso).

Il quadro della percezione popolare delle lentiggini si completa infine con il detto paremiaco «sotto una lentiggine c'è una bella ragazza», declinato ad esempio nelle varianti dialettali monferrina sota na lantija j'è na bela fija (Culasso \& Viberti, 2003), ligure sut a lentigja ke sta a bela figja (AIS: P. 169 n.) e triestina soto una lentìa se scondi 'na bela fia (Pinguentini, 1969) che sembrerebbero suggerire una valutazione positiva delle lentiggini; ma, come spesso accade nel campo della paremiologia, la forma ampliata del medesimo proverbio, ovvero suta na lentia j'è na bela fija, pasà ed tre bruta l'è, pasa ed sent fa pau a la gènt «sotto una lentiggine c'è una bella ragazza, se ne ha tre brutta è, sopra le cento fa paura alla gente!» restituisce una visione negativa che collima con quella trasmessa dalla medicina popolare e riflessa, come si è visto, in alcune denominazioni dialettali.

\section{RIFERIMENTI BIBLIOGRAFICI}

AIS $=$ JABERG Karl \& JuD Jakob, 1928-1940, Sprach- und Sachatlas Italiens und der Südschweiz, 8 voll., Zofingen, Ringier u. C. (anche <www3.pd.istc.cnr.it/ navigais-web/>).

ALD II = Goebl Hans (éd.), 2012, Atlante linguistico del ladino dolomitico e dei dialetti limitrofi, $2^{\text {a }}$ parte, 5 voll., Strasbourg, Éditions de linguistique et de philologie.

ALEIC = BotTIGLIONI Gino, 1933-1942, Atlante linguistico etnografico italiano della Corsica, Pisa.

5. <www.leviedellasardegna.eu/la_medicina_popolare_in_sardegna.html>. 
ALI = BARToli Matteo et al., 1995-, Atlante Linguistico Italiano, I-, Roma, Istituto Poligrafico e Zecca dello Stato.

$\mathrm{ALT}=$ Atlante Lessicale Toscano-ALTWeb, $<\mathrm{http}: / /$ serverdbt.ilc.cnr.it/altweb/>.

Altamura Antonio, 1956, Dizionario dialettale napoletano, Napoli, Fiorentino.

ANDREOli Raffaele, 1887, Vocabolario napoletano-italiano, Paravia.

ASLEF = Pellegrini Giovan Battista, 1972-1986, Atlante Storico-LinguisticoEtnografico Friulano, 6 voll., Istituto di Glottologia dell'Università di Padova, Istituto di filologia romanza della Facoltà di Lingue e letterature straniere di Trieste con sede a Udine.

Battipede Benedetto, 1987, Dizionario del dialetto di Castrovillari, Castrovillari, ed. Il Coscile.

Bianchini Giovanni \& BRacchi Remo, 2003, Dizionario etimologico dei dialetti della Val Tartano, Sondrio.

Boerio Giuseppe, $1856^{2}$, Dizionario del dialetto veneziano, Venezia, Cecchini.

Brighenti Eliseo, 1927, Dizionario greco moderno-italiano e italiano greco-moderno, Milano, Hoepli.

Cortelazzo Manlio, 2007, Dizionario veneziano della lingua e della cultura popolare nel XVI secolo, Padova, La Linea Editrice.

Croatto Enzo, 2004, Vocabolario del dialetto ladino-veneto della Valle di Zoldo (Belluno), Vicenza, Regione del Veneto - Angelo Colla Editore.

Crusca, 1863-1923 = Vocabolario degli accademici della Crusca (<www.lessicografia.it>).

Culasso Primo \& Viberti Silvio, 2003, Rastlèire. Vocabolàri d'Arba, Langa e Roé, Savigliano, Gribaudo.

D’Ambra Raffaele, 1873 [1969], Vocabolario napolitano-toscano, Bologna, Forni.

DAM = GiAMMARCo Ernesto, 1968-1979, Dizionario abruzzese-molisano, 4 voll., Roma, Edizioni dell'Ateneo.

DEI = BatTISTI Carlo \& Alessio Giovanni, 1950-1957, Dizionario etimologico italiano, 5 voll., Firenze, Barbera.

DELI = Cortelazzo Manlio \& Zolli Paolo, 1980, Dizionario etimologico della lingua italiana, Bologna, Zanichelli.

DES = WAGNER Max Leopold, 1962, Dizionario etimologico sardo, 4 voll., Heidelberg, Winter Universitätsverlag.

ERCOlAni Libero, 1994, Nuovo vocabolario romagnolo-italiano, italiano-romagnolo, Ravenna, Ed. del Girasole.

EVLI = Nocentini Alberto, 2010, L'Etimologico. Vocabolario della lingua italiana, Le Monnier.

FEW = VON WARTBURG Walter, Französisches etymologisches Wörterbuch, Bonn, 1922-1928; Leipzig, 1932-1940; Basel, 1944-.

Forni Marco, Dizionario on line Italiano - Ladino della Val Gardena / Ladino della Val Gardena - Italiano, <http://dizionario-italiano-gardenese.ladinternet. it $/>$.

LEA = GiAMMARCo Ernesto, 1985, Lessico etimologico abruzzese, Roma, Edizioni dell'Ateneo. 
LEI = PFISTER Max et al., 1979-, Lessico etimologico italiano, Wiesbaden, Reichert. Maragliano Alessandro, 1962, Tradizioni popolari vogheresi, Firenze, Le Monnier. Menegus Tamburin Vincenzo, 1973, Dizionario del dialetto di Cortina d'Ampezzo, Vicenza, Neri Pozza.

Menegus Tamburin Vincenzo, 1978, Il dialetto nei paesi cadorini d'Oltrechiusa, Firenze, Istituto di Studi per l'Alto Adige.

Miniati Valeria, 2010, Italiano di Romagna. Storia di usi e di parole, Bologna, Clueb.

Monti Pietro, 1848, Vocabolario dei dialetti della città e diocesi di Como, Bologna, Forni.

NDDC $=$ RohlFs Gerhard, 1977, Nuovo dizionario dialettale della Calabria, Ravenna, Longo Editore.

Pallabazzer Vito, 1988, Lingua e cultura ladina: lessico e onomastica di Laste, Rocca Pietore, Colle S. Lucia, Selva di Cadore, Alleghe, Belluno, Istituto bellunese di ricerche sociali e culturali.

PAZZINI Adalberto, 1948, La medicina popolare in Italia, ed. Zigiotti.

Pinguentini Gianni, 1969, Nuovo dizionario del dialetto triestino, Bologna, Cappelli Editore.

Pirona Giulio Andrea, 1935, Il nuovo Pirona, vocabolario friulano, Udine, Arturo Bosetti Editore.

Pitré Giuseppe, 1896, Medicina popolare siciliana, Torino/Palermo, Clausen.

REP = CoRnAGLIOTTI Anna et al., 2015, Repertorio etimologico piemontese, Torino, Centro di Studi Piemontesi.

REW = MEYER-LÜBKE Wilhelm, 1935, Romanisches etymologisches Wörterbuch, Heidelberg.

REWS = FARÉ Paolo A., 1972, Postille italiane al Romanisches etymologisches Wörterbuch, Milano, Istituto Lombardo di Scienze e Lettere.

RoHLFs Gerhard, 1966-1969, Grammatica storica della lingua italiana e dei suoi dialetti, 3 voll., Torino, Einaudi.

Vallebona Giuseppe, 1980, Dizionario Tabarkino-Italiano, Genova, Compagni dei Librai.

VDS = RoHLFs Gerhard, 1956-1961, Vocabolario dei dialetti salentini (Terra d'Otranto), München, Bayerische Akademie der Wissenschaften.

VS = Piccitтo Giorgio, 1977, Vocabolario siciliano, Catania-Palermo, Centro di studi filologici e linguistici siciliani. 